Herz 2018 4 43:483

https://doi.org/10.1007/s00059-018-4724-0

(c) Springer Medizin Verlag GmbH, ein Teil von Springer Nature 2018

CrossMark

\title{
R. Zahn
}

Med. Klinik B: Kardiologie, Pneumologie, Internistische Intensivmedizin, Klinikum Ludwighafen, Ludwigshafen, Deutschland

\section{Strukturen in der Kardiologie}

Normalerweise werden in der Zeitschrift Herz aktuelle Themen der Diagnostik und Therapie von Herzerkrankungen behandelt. Manchmal werden dabei auch Grundlagenthemen mitbesprochen oder stehen gar im Zentrum. Die vorliegende Ausgabe macht hier eine Ausnahme: Unser Alltag, sowohl in der Klinik wie auch in der Praxis, wird nicht nur von der Diagnostik und Behandlung von Patienten bestimmt, sondern zunehmend auch von Strukturen (Qualitätssicherungsmaßnahmen, Hygienevorschriften, ...), die uns häufig von außen (Klinikleitung, Gesetzgeber etc.) auferlegt werden. Manche dieser Strukturen stammen aber auch von unseren eigenen Fachgesellschaften und sollen unter anderem in Zeiten eines rasch fortschreitenden Erkenntniszuwachses bestimmte Standards in Diagnostik und Therapie etablieren. Inwieweit diese Strukturen aber in den Klinikalltag passen, wie sie mit der Weiterbildungsberechtigung harmonisieren und etablierte klinikinterne Leitungsstrukturen in Frage stellen, bleibt oft unberücksichtigt. Diesem Spannungsfeld widmet sich das vorliegende Heft.

In dem ersten Artikel von Halbach und Baldus werden die positiven Aspekte von Zertifizierungen in der Kardiologie dargelegt, während der Kontra-Artikel von Schächinger et al. auf Probleme von Zertifizierungen, sowohl auf solche methodischer Natur als auch auf deren Integration in bestehende Strukturen, hinweist und ein mehr integratives Konzept und mehr Abstimmung fordert.

Die folgenden 3 Artikel beschäftigen sich mit folgenden Einzelthemen: Frau Semmler behandelt die Morbiditäts- und Mortalitätskonferenzen, ihren Aufbau und ihre Funktion in einem Qualitätskonzept einer Klinik. Die gerade neu generierten Cardiac-Arrest-Zentren werden von Scholz und Böttiger vorgestellt und ihr Sinn und ihre Strukturen besprochen. Frau Güder und Herr Ertl besprechen die Herzinsuffizienznetzwer$k e$, wie sie aussehen sollen und was sich bereits etabliert hat.

Ich hoffe, mit dem vorliegenden Heft über ein ungewohntes Thema Ihr Interesse für Strukturen in der Kardiologie geweckt und Anregungen für eine Diskussion geliefert zu haben. Ich denke, es muss in unserem Sinne sein, Strukturen selbst mitzugestalten, anstatt nur unter ihnen zu leiden.

\section{R. Zahn}

\section{Korrespondenzadresse}

Prof. Dr. R. Zahn

Med. Klinik B: Kardiologie, Pneumologie, Internistische Intensivmedizin, Klinikum Ludwighafen

Bremserstr. 73, 67063 Ludwigshafen,

Deutschland

erzahn@aol.com

Interessenkonflikt. R. Zahn gibt an, dass kein Interessenkonflikt besteht. 